\title{
Preisach modeling of temperature-dependent ferroelectric response of piezoceramics at sub- switching regime
}

\author{
Diego Alejandro Ochoa, Jose Eduardo García* \\ Department of Applied Physics, Universitat Politècnica de Catalunya - BarcelonaTech \\ 08034 Barcelona, Spain
}

\begin{abstract}
The Preisach model is a classical method for describing nonlinear behavior in hysteretic systems. According to this model, a hysteretic system contains a collection of simple bistable units which are characterized by an internal field and a coercive field. This set of bistable units exhibits a statistical distribution that depends on these fields as parameters. Thus, nonlinear response depends on the specific distribution function associated with the material. This model is satisfactorily used in this work to describe the temperature-dependent ferroelectric response in PZT- and KNN-based piezoceramics. A distribution function expanded in Maclaurin series considering only the first terms in the internal field and the coercive field is proposed. Changes in coefficient relations of a single distribution function allow us to explain the complex temperature dependence of hard piezoceramic behavior. A similar analysis based on the same form of the distribution function shows that the KNL-NTS properties soften around its orthorhombic to tetragonal phase transition.
\end{abstract}

Keywords: PZT; ceramics; dielectric properties; ferroelectrics; Preisach model.

\footnotetext{
${ }^{*}$ Corresponding author. Tel.: +34 934016086; fax: +34 934016090.

E-mail address: jose.eduardo.garcia@upc.edu (J.E. Garcia).
} 


\section{Introduction}

Piezoelectric materials are used in a broad range of applications including, for instance, sonars, ultrasonic cleaners, buzzers, accelerometers, transformers, ultra-precision positioners and ultrasonic motors. The most widely used piezoelectric materials are lead-based ferroelectric ceramics. Piezoelectric ceramics based on lead zirconate titanate system, $\mathrm{Pb}(\mathrm{Zr}, \mathrm{Ti}) \mathrm{O}_{3}(\mathrm{PZT})$, currently dominate the market of piezoceramic applications due to their excellent functional properties [1]. However, since PZT materials contain more than $60 \mathrm{wt} . \%$ lead, they pose a potential ecological hazard. Given the need for environmental protection, lead-free piezoelectric ceramics are receiving great attention as environmentally friendly materials [2]. In particular, much attention has been paid to $(\mathrm{K}, \mathrm{Na}) \mathrm{NbO}_{3}(\mathrm{KNN})$ ceramic systems because of their relatively strong piezoelectric properties and high Curie temperature [3].

Compositional modifications by doping are extensively used to tune functional properties of piezoceramics for specific applications. Thus, piezoceramics are classified into two main groups, hard and soft, according to their improved properties [1]. For instance, soft piezoceramics exhibit high piezoelectric constants but high losses, while hard piezoceramics display low losses and a high quality factor, but low piezoelectric constants. Hard PZT is obtained through acceptor (hardener) substitutions, whereas soft PZT is obtained by means of donor (softener) substitutions, e.g. the substitution of $\mathrm{Zr}^{4+}$ or $\mathrm{Ti}^{4+}$ by trivalent (like $\mathrm{Fe}^{3+}$ ) or pentavalent (like $\mathrm{Nb}^{5+}$ ) cations, respectively. The addition of dopants determines the extrinsic response of the material, with the result that not only the values of hard and soft material properties are different but also their behavior (i.e. the property-dependence with temperature [4], electric field [5] and/or mechanical stress [6] are notably different for hard and soft piezoceramics). Compositional engineering has also been the key to obtain what is probably the most workable lead-free composition known to date: the $\left(\mathrm{K}_{0.44} \mathrm{Na}_{0.52} \mathrm{Li}_{0.04}\right)\left(\mathrm{Nb}_{0.86} \mathrm{Ta}_{0.10} \mathrm{Sb}_{0.04}\right) \mathrm{O}_{3}$ (KNL-NTS) piezoceramic [7]. KNL-NTS obtained by conventional processing exhibits a good longitudinal piezoelectric coefficient $(>250 \mathrm{pC} / \mathrm{N})$ and high Curie temperature $\left(\sim 250^{\circ} \mathrm{C}\right)$. This material has a typical soft behavior exemplified by high dielectric losses, low quality factor, 
This is the post-print (i.e. final draft post-refereeing) of the publication.

The final publication is available at Springer via http://dx.doi.org/ 10.1007/s00339-016-9808-1

'square' hysteresis loop, and properties strongly dependent on the applied electric field and/or mechanical stress [8].

Piezoelectric devices are required to work in various environments. As a consequence, knowledge of the material properties under the specific working conditions is crucial for the design of piezoelectric devices. Most notably, this includes the temperature of piezoelectric devices during operation, which can range from hot, as in combustion engines, to very cold, as those occurring in aerospace applications. The temperature variation of the properties of the piezoceramics reveals that the material properties, and consequently the performance of the devices at the working environment, can differ significantly from those at ambient temperature. From this perspective, understanding and modeling the temperature dependence of functional properties of piezoceramics is an important question that must be solved in order to improve the design of piezoelectric devices. The Preisach model is used in this work in order to model the thermal evolution of the extrinsic response behavior of PZT (lead-based) and KNN (lead-free) piezoceramic compositions at sub-switching regime.

\section{Theoretical considerations}

The Preisach model is a classical method for describing nonlinear behavior in hysteretic systems and has been used satisfactorily in ferroelectrics [9-12]. According to this model, a hysteretic system contains a collection of simple bistable units (Fig. 1(a)) with both states contributing independently to the total response by the same amount $\pm P_{0}$. These units are characterized by an internal field $E_{i}$, and a coercive field $E_{c}$, so that the set of bistable units exhibits a statistical distribution $f\left(E_{i}, E_{c}\right)$ that depends on the parameters $-\infty<E_{i}<\infty$ and $0<E_{C}<\infty$. The response to an applied external field depends on both the amount of bistable units switched and the direction of applied field change. For increasing fields $\left(E_{\text {inc }}\right)$, when $E_{\text {inc }}>E_{i}+E_{C}$, the bistable units in the negative state of polarization $\left(-P_{0}\right)$ switch to the positive state $\left(+P_{0}\right)$, each one contributing by $2 P_{0}$ to the total response (Fig. 1(b)). For decreasing fields, when 
$E_{\text {dec }}<E_{i}-E_{C}$, each unit in the state $+P_{0}$ contributes by $-2 P_{0}$ to the total response switch to the state $-P_{0}$ (Fig. 1(b)). So, if the applied field is an alternative field $E(t)=E_{0} \cos \omega t$, where $E_{0}$ is the amplitude of the applied alternative electric field, then according to whether the applied field increases (Fig. 1(c)) or decreases (Fig. 1(d)), the bistable units inside the grey triangular regions of Preisach plane contributing to the total response switch to their opposite state [13].
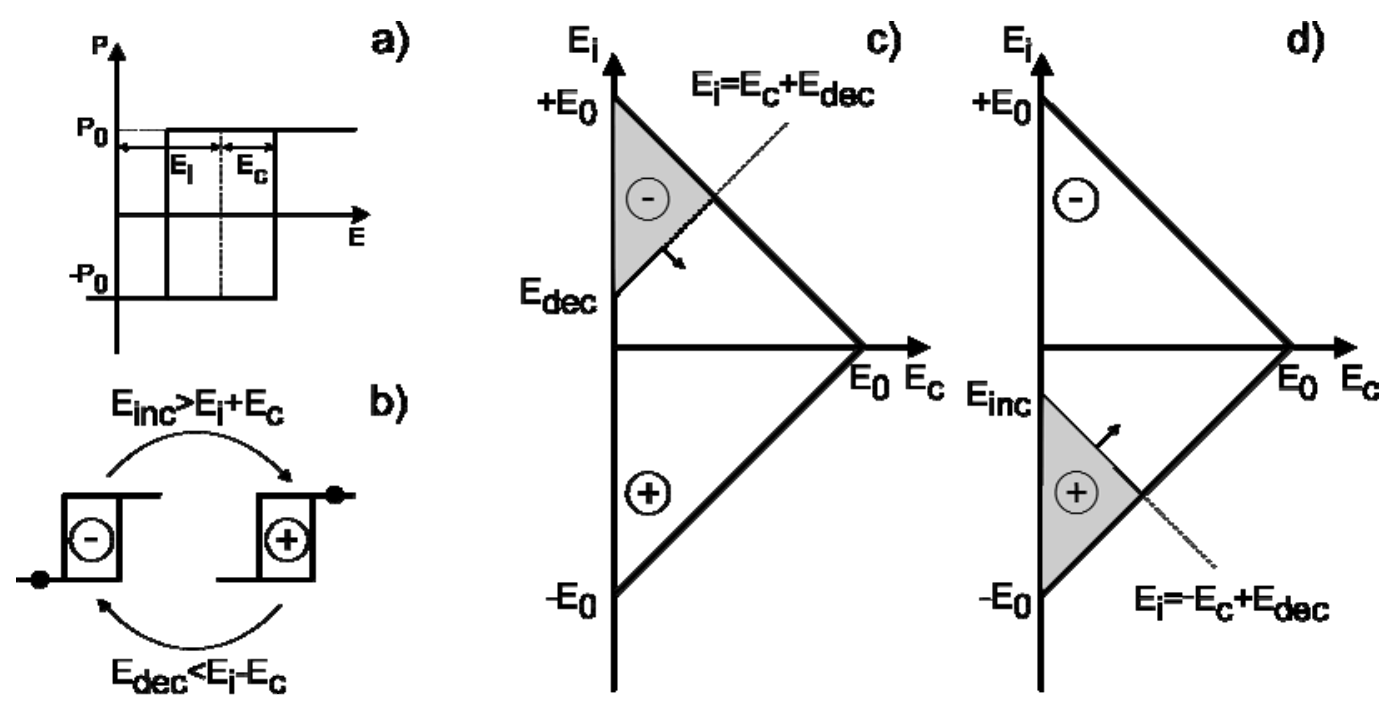

Fig. 1 Schematic representation of the bistable unit and the Preisach plane: a) the bistable unit showing its characteristic parameters; $b$ ) conditions fulfilled for the up or down switching of the bistable unit; c) bistable elements that are switched when the field goes down; d) bistable elements that are switched when the field goes up.

The branches of the hysteresis loop due to the nonlinear behavior of the system can be obtained from the field-dependent integrals of the distribution function over the grey triangular regions given in Figs. 1(c) and 1(d):

$$
\begin{gathered}
D^{-}(E)=\frac{D_{N L}\left(E_{0}\right)}{2}-2 P_{0} \int_{0}^{\left(E_{0}-E\right) / 2} \int_{E+E_{c}}^{E_{0}-E_{c}} f\left(E_{i}, E_{c}\right) d E_{i} d E_{c}, \\
D^{+}(E)=-\frac{D_{N L}\left(E_{0}\right)}{2}+2 P_{0} \int_{0}^{\left(E_{0}+E\right) / 2} \int_{-E_{0}+E_{c}}^{E-E_{c}} f\left(E_{i}, E_{c}\right) d E_{i} d E_{c} ;
\end{gathered}
$$

which is 
This is the post-print (i.e. final draft post-refereeing) of the publication.

The final publication is available at Springer via http://dx.doi.org/ 10.1007/s00339-016-9808-1

$$
D_{N L}\left(E_{0}\right)=2 P_{0} \int_{0}^{E_{0}} \int_{-E_{0}+E_{c}}^{E_{0}-E_{c}} f\left(E_{i}, E_{c}\right) d E_{i} d E_{c} .
$$

Eq. (1) corresponds to the descending branch of the loop (Fig. 1(c)), where the bistable units change from a positive to a negative state of polarization. Eq. (2) is the ascending branch (Fig. 1(d)), where the bistable units change from a negative to a positive state. The quantity $D_{N L}\left(E_{0}\right)$, which represents the total nonlinear contribution, depends on the number of switched units inside the triangular region of Preisach plane. The term $\left( \pm D_{N L} / 2\right)$ is added to the integrals in Eqs. (1) and (2) because the extreme values of each loop branch must coincide. In addition, it is also useful to centre the hysteresis loops.

The hysteretic nonlinear response can be rewritten as the sum of two terms that embody the two branches of the loop:

$$
D_{N L}(E)=\bar{D}(E) \pm \Delta D(E),
$$

being

$$
\bar{D}(E)=\frac{D^{+}(E)+D^{-}(E)}{2}
$$

and

$$
\Delta D(E)=\frac{D^{+}(E)-D^{-}(E)}{2}
$$

The simplest Preisach model development corresponds to a flat uniform distribution function $f\left(E_{i}, E_{c}\right)=g_{0}$. The branches of the sub-switching hysteresis loop can be obtained by substituting this distribution function in the Eqs. (1) and (2). Solving the integrals and evaluating Eqs. (5) and (6), we obtain $D_{N L}$ in the form given by Eq. (4):

$$
D_{N L}=P_{0} g_{0} E_{0} E \pm \frac{1}{2} P_{0} g_{0}\left(E_{0}^{2}-E^{2}\right) .
$$

Eq. (7) matches the Rayleigh law for describing the nonlinear sub-switching ferroelectric response [14]. This empirical model is widely accepted in the literature for 
This is the post-print (i.e. final draft post-refereeing) of the publication.

The final publication is available at Springer via http://dx.doi.org/ 10.1007/s00339-016-9808-1

describing the nonlinear behavior of soft piezoceramics [15-17]. It has recently been shown that soft PZT response can be described by the Rayleigh model over a wide range of temperatures [18]. Therefore, the temperature-dependent ferroelectric response of soft piezoceramics at the sub-switching regime could be described by using the Preisach model with a flat distribution function in which $g_{0}$ depends on the temperature, $f\left(E_{i}, E_{c}\right)=g_{0}(T)$.

Hard piezoceramics, on the other hand, show a different and more complex behavior. The main observable characteristic of these types of materials is that the major hysteresis loop appears pinched, which leads to a drastic decrease in the remanent polarization [19]. Hard PZT behavior has been described in the literature by using the Preisach model with a fourth order polynomial distribution function, symmetric in terms of $E_{i}$, combined with a cut-off at high coercive fields. However, the same reference discusses how a more realistic distribution may be obtained by two superimposed Gaussians, symmetrical with respect to $E_{i}$ [9]. More general distribution functions, based in the shape of major hysteresis loop and its evolution under external actions (e.g. mechanical stress), have been proposed by other authors [20-23]. In all instances, when the applied electric fields are sufficiently below the coercive field (i.e. subswitching regime), the distribution function may be expanded in a Maclaurin series [13] considering only the first terms in $E_{i}$ and $E_{C}$. Taking into account the temperature dependence of the ferroelectric response, it is possible to assume a temperature dependence of the function coefficients:

$$
f\left(E_{i}, E_{c}\right)=g_{0}(T)+g_{2}(T) E_{i}^{2}+g_{1}^{C}(T) E_{C} .
$$

It should be noted that although a flat distribution may describe the soft piezoceramic behavior, the function distribution given in Eq. (8) can also describe this behavior by assuming the contribution of $g_{0}(T)$ to be higher than the contribution of the other terms. In order to perform a general analysis, we use Eq. (8) as a general distribution function for both hard and soft piezoceramics. Thus, we can obtain one general mathematical expression for each branch of 
the sub-switching hysteresis loop by solving Eqs. (1) and (2) for the function distribution given in Eq. (8). From $D^{+}$and $D^{-}$, we can obtain $\bar{D}$ (Eq. (5)) as follows:

$$
\bar{D}=\left[\frac{P_{0}}{12}\left(g_{2}(T) E_{0}+g_{1}^{C}(T)\right)\right] E^{3}+\left[\frac{P_{0}}{12} g_{2}(T) E_{0}^{3}+\frac{P_{0}}{12} g_{1}^{C}(T) E_{0}^{2}+P_{0} g_{0}(T) E_{0}\right] E
$$

Note that for $g_{i \neq 0}=0$, Eq. (9) returns to the result given for a flat distribution, $\bar{D}=P_{0} g_{0} E_{0} E$, for which a linear dependence is revealed. For simplicity, we can rewrite Eq. (9) as follows:

$$
\bar{D}=a_{3}\left(E_{0}, T\right) E^{3}+a_{1}\left(E_{0}, T\right) E,
$$

where

$$
a_{1}=\frac{P_{0}}{12} g_{2}(T) E_{0}^{3}+\frac{P_{0}}{12} g_{1}^{C}(T) E_{0}^{2}+P_{0} g_{0}(T) E_{0}
$$

and

$$
a_{3}=\frac{P_{0}}{12}\left(g_{2}(T) E_{0}+g_{1}^{C}(T)\right) \text {. }
$$

The coefficients $a_{1}$ and $a_{3}$ depend on the temperature and the amplitude of the electric field. To analyze the preponderant behavior to material response, it is necessary to compare the contribution of both terms in Eq. (10). The coefficients $a_{1}$ and $a_{3}$ cannot be compared numerically because they are different physical magnitudes. However, we can define a threshold field

$$
E_{t h}=\sqrt{\frac{a_{1}}{a_{3}}}
$$

as the value for which both cubic and linear terms contribute equally to material response. If the applied electric field is higher than the threshold field $\left(E>E_{t h}\right)$ the dominant behavior is cubic, otherwise the dominant behavior is linear if the applied electric field is lower than the threshold field $\left(E<E_{t h}\right)$. A linear behavior $\left(E_{t h}>>E\right)$ is predicted by the Rayleigh model for $\bar{D}(E)$ function that corresponds to a soft behavior. Therefore, a hard behavior should be revealed depending on whether the cubic term in Eq. (10) is of the order of or greater than the linear term, i.e. from $E_{\text {th }} \approx E$ up to $E_{\text {th }}<<E$. 
This is the post-print (i.e. final draft post-refereeing) of the publication.

The final publication is available at Springer via http://dx.doi.org/ 10.1007/s00339-016-9808-1

\section{Experimental procedure}

$\mathrm{Nb}$ - (soft PZT) and Fe-doped (hard PZT) $\mathrm{PbZr}_{0.60} \mathrm{Ti}_{0.40} \mathrm{O}_{3}$ powders were prepared by solid state reaction. The powders were uniaxially pressed at $100 \mathrm{MPa}$ into pellets of $20 \mathrm{~mm}$ in diameter and about $2 \mathrm{~mm}$ thickness, and then isostatically pressed at $120 \mathrm{MPa}$. Sintering was carried out in a saturated $\mathrm{PbO}$ atmosphere at 1200 or $1250{ }^{\circ} \mathrm{C}$, according to the PZT composition. $\left(\mathrm{K}_{0.44} \mathrm{Na}_{0.52} \mathrm{Li}_{0.04}\right)\left(\mathrm{Nb}_{0.86} \mathrm{Ta}_{0.10} \mathrm{Sb}_{0.04}\right) \mathrm{O}_{3}$ ceramics $(\mathrm{KNL}-\mathrm{NTS})$ was also prepared by solid state reaction. The raw powders were weighed according to the stoichiometric ratio and were attrition-milled using $\mathrm{ZrO}_{2}$ balls in ethanol medium for $3 \mathrm{~h}$, then dried and calcined at $700{ }^{\circ} \mathrm{C}$ for $2 \mathrm{~h}$. The calcined powders were attrition-milled again and pressed at $200 \mathrm{MPa}$ into pellets of $20 \mathrm{~mm}$ diameter and $2 \mathrm{~mm}$ thickness. Sintering was carried out at $1125{ }^{\circ} \mathrm{C}$ for $16 \mathrm{~h}$ in air atmosphere. Room temperature X-ray diffraction analysis revealed the expected rhombohedral crystallographic phase for PZT-based materials and the tetragonal crystallographic phase for KNN-based compound. Samples were cut into discs of $\sim 15 \mathrm{~mm}$ in diameter and $\sim 1 \mathrm{~mm}$ thickness. After polishing the samples, silver electrodes were painted on both sides of the discs. Nonlinear measurements were performed in a closed-loop cryostat over a wide temperature range using a capacitance comparator bridge specially designed for this type of measurement [24]. A sinusoidal electric field of $1 \mathrm{kHz}$ frequency is applied to the samples. The instantaneous nonlinear current was measured as a function of the instantaneous applied electric field. The electrical displacement $D_{N L}(E)$ was obtained by numerical integration of the current for each value of the electric field amplitude. In order to analyse the non-dissipative contribution to $D_{N L}$, i.e. the first term $(\bar{D})$ of Eq. (4), the arithmetic average (half-sum) of $D_{N L}$ for each instantaneous value of the field is computed. The dielectric losses are directly related with the second term ( $\Delta D)$ of Eq. (4), which represents the dissipative contribution. It is computed as the half-difference of the two values of $D_{N L}$ that are associated to each value of electric field (see Fig. 2). 
This is the post-print (i.e. final draft post-refereeing) of the publication.

The final publication is available at Springer via http://dx.doi.org/ 10.1007/s00339-016-9808-1

\section{Results and discussion}

\subsection{PZT-based piezoceramics}

Fig. 2 shows the nonlinear sub-switching hysteresis loop of soft (Fig. 2(a)) and hard (Fig. 2(b)) PZT for an amplitude of the applied electric field of $\sim 0.6 \mathrm{kV} / \mathrm{mm}$ at room temperature. The function $\bar{D}(E)$ that represents the non-dissipative contribution is also plotted for both materials. The insets show the dissipative contribution $\Delta D$ as a function of the instantaneous value of the electric field. Remarkable differences are displayed when the behavior of both materials are compared. A Rayleigh-type sub-switching hysteresis loop that can be described by Eq. (7) is observed for soft PZT. In this case, the function $\bar{D}(E)$ seems to follow a linear dependence, while $\Delta D(E)$ appears to show a quadratic behavior. Conversely, in hard PZT, $\bar{D}(E)$ exhibits a nonlinear behavior and $\Delta D(E)$ cannot be described by a quadratic function.

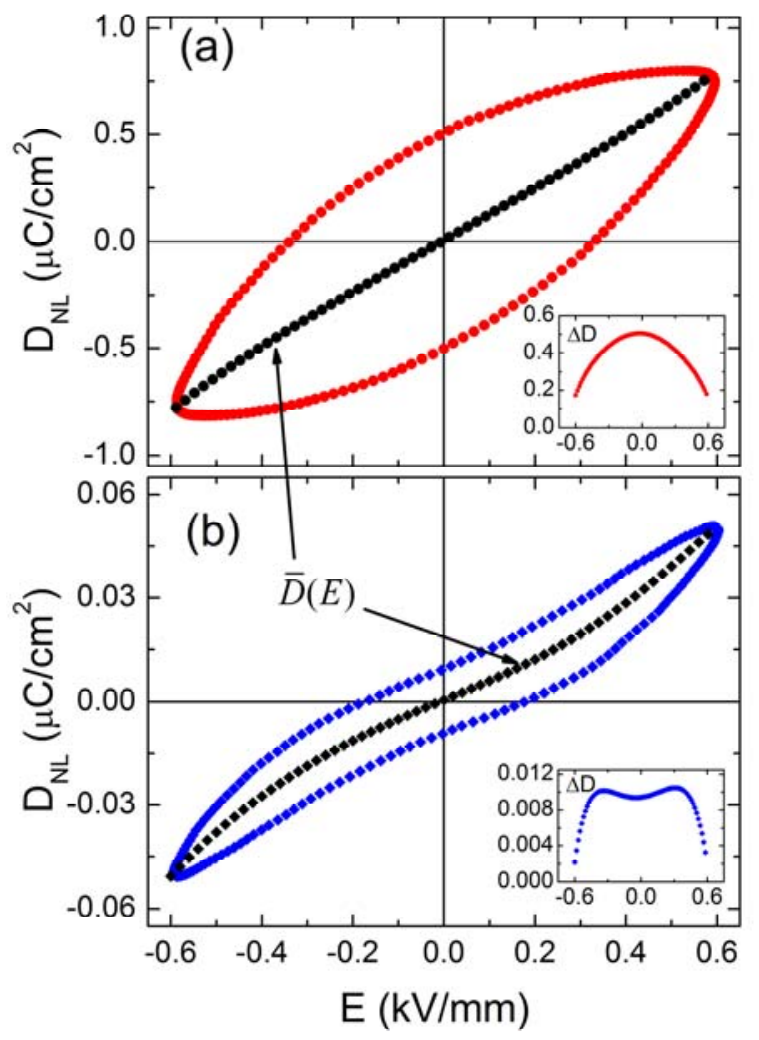

Fig. 2 Nonlinear sub-switching hysteresis loop of soft (a) and hard (b) PZT for an amplitude of the applied electric field of $\sim 0.6 \mathrm{kV} / \mathrm{mm}$ at $1 \mathrm{kHz}$ frequency and at room temperature $(\sim 300 \mathrm{~K})$. The half-sum of $D_{N L}$ for each instantaneous value of the field, $\bar{D}$, is plotted for both materials. The insets show the respective half-difference, $\Delta D$, of the two values of $D_{N L}$ as a function of the electric field. 
This is the post-print (i.e. final draft post-refereeing) of the publication.

The final publication is available at Springer via http://dx.doi.org/ 10.1007/s00339-016-9808-1

Assuming the theoretical proposal based on a unique distribution function (i.e. Eq. (8)), the non-dissipative contribution should match the general behavior given in Eq. (10) for both soft and hard piezoceramics. In this context, the linear fitting coefficient $a_{1}$ is expected to dominate soft behavior, while the cubic fitting coefficient $a_{3}$ should become significant for describing hard behavior. The values of $a_{1}$ and $a_{3}$ are computed over a wide temperature range by fitting the function $\bar{D}(E)$ to Eq. (10). Good fitting parameters are reached in all cases $\left(R^{2} \geq 0.9999\right)$. The absolute values of the fitting coefficients cannot assess what is the preponderant behavior for any of materials at a given temperature. Therefore, the threshold field $E_{t h}$, defined in Eq. (13), is calculated to effectively compare the contribution of both linear and cubic terms to $\bar{D}$.

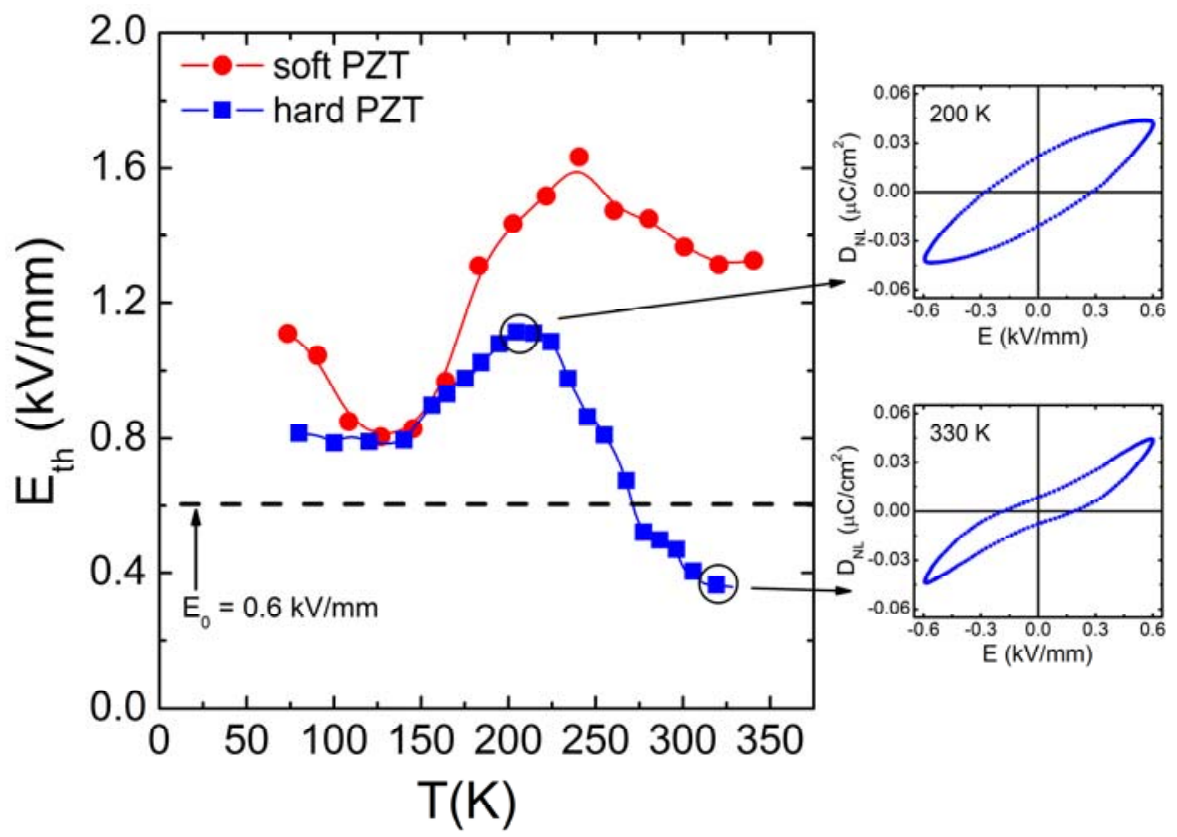

Fig. 3 Temperature dependence of the threshold field for soft and hard PZT. The horizontal dashed line represents the maximum value (the amplitude) of the applied electric field, $E_{0}=0.6$ $\mathrm{kV} / \mathrm{mm}$. The lines that link $E_{\text {th }}$ values are only a guide for the eyes. For purposes of comparison, sub-switching hysteresis loops are shown for two different temperatures.

Fig. 3 shows $E_{t h}$ as a function of the temperature for both soft and hard PZT. It is shown that $E_{\text {th }}$ is higher than the electric field amplitude, $E_{0}$, in soft PZT for all temperatures, 
which makes a linear behavior for $\bar{D}(E)$ function evident in this case. Consequently, soft-type behavior is verified in soft PZT across the whole temperature range. On the other hand, two regions of $E_{t h}$ values can be glimpsed in hard PZT, i.e. $E_{t h}>E_{0}$ at temperatures below $\sim 275 \mathrm{~K}$ and $E_{\text {th }}<E_{0}$ at temperature above $\sim 275 \mathrm{~K}$. Each of these regions is characterized by a different dominant contribution. Thus, a linear behavior for $\bar{D}(E)$ function is also displayed in hard PZT at low temperatures. In Fig 3, the sub-switching hysteresis loops at $200 \mathrm{~K}$ and $320 \mathrm{~K}$ are shown in order to illustrate the behavior change. This soft-to-hard behavior change in hard PZT has been experimentally revealed in a recent work by means of the temperature dependence nonlinear dielectric response and hysteresis loop measurements. This fact, now corroborate by using the Preisach model, was explained as a manifestation of a change in the domain wall dynamic [18].

\subsection{KNN-based piezoceramics}

The threshold field, $E_{t h}$, is also calculated for KNL-NTS piezoceramics over a wide temperature range and is shown in Fig. 4. As can be observed, $E_{t h}$ is higher than $E_{0}$ for all temperatures, which indicates a linear behavior for $\bar{D}(E)$ function. Hence, soft-type behavior is revealed for this compound at any temperature in the testing range.

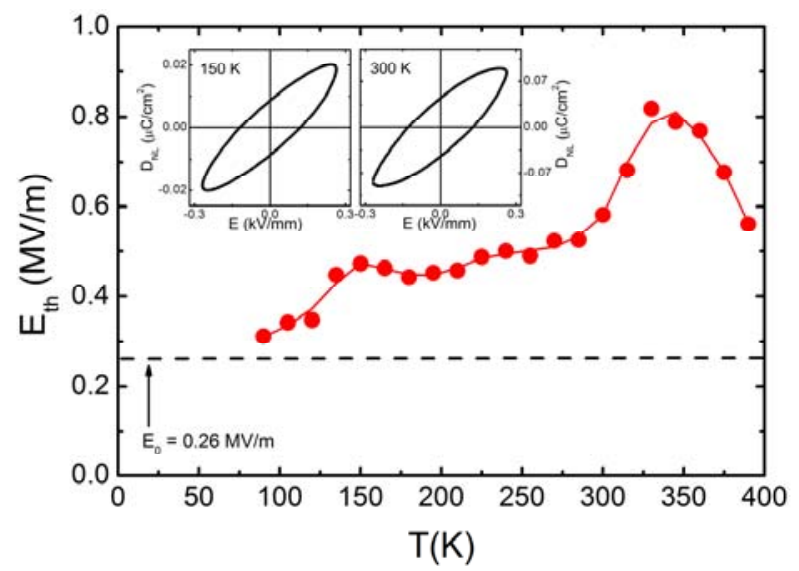

Fig. 4 Temperature dependence of the threshold field for KNL-NTS piezoceramics. The horizontal dashed line represents the maximum value (the amplitude) of the applied electric field, $E_{0}=0.26 \mathrm{kV} / \mathrm{mm}$. The lines that link $E_{t h}$ values are only a guide for the eyes. For purposes of comparison, sub-switching hysteresis loops are shown for two different temperatures. 
Although $E_{t h}>E_{0}$ for all temperatures in KNL-NTS, an interesting feature is displayed in $E_{t h}(T)$ function for this material, and that is that a maximum appears at $\sim 325 \mathrm{~K}$. Note that as $E_{\text {th }}$ becomes larger, the linear dependence of the $\bar{D}(E)$ function becomes more definite, i.e. the softer is the material behavior. It is well-known that KNL-NTS exhibits an orthorhombic to tetragonal phase transition near to room temperature [8]. Therefore, the ferroelectric-toferroelectric phase transition seems to soften the material response.

\section{Conclusions}

The Preisach model has been satisfactorily used to describe the temperature dependence of ferroelectric response of piezoceramics at sub-switching regime. A simple distribution function based on the Maclaurin series expansion is used to model both soft and hard behaviors. The model fits with the experimental measurements by using the same form of the distribution function, which accounts for the different observed behaviors. Temperature-dependent coefficients are taken into consideration in order to evaluate the ferroelectric response of the studied materials over a wide temperature range. Soft-type behavior is confirmed for soft PZT for all temperatures, while a transition from soft- to hard-type behavior is detected for hard PZT at $\sim 275 \mathrm{~K}$. This transition seems to be associated to a change in dynamics of the domain wall motion. The same distribution function is used to model the temperature dependence of ferroelectric behavior of the KNL-NTS piezoceramic. It is revealed for the first time that this material exhibits a soft-type behavior across all the temperature range. The Preisach approach shows that the material properties soften near a ferroelectric-to-ferroelectric phase transition. This outcome could be significant for designing new materials for specific applications, e.g. when very high values of dielectric constant and/or piezoelectric coefficient are necessary, and when the possible high losses and properties instability do not affect device operation. 
This is the post-print (i.e. final draft post-refereeing) of the publication.

The final publication is available at Springer via http://dx.doi.org/ 10.1007/s00339-016-9808-1

Acknowledgments This work is supported by the MINECO project MAT2013-48009-C4-2-P of the Spanish Government. The authors wish to thank to Prof. Jose A. Eiras, at the Universidade Federal de São Carlos, Brazil, and Prof. Jose F. Fernández, at the Instituto de Cerámica y Vidrio - CSIC, Spain, for providing the samples. The authors also gratefully acknowledge Dr. Raúl Cruz Hidalgo, at the Universidad de Navarra, for his useful discussions.

\section{References}

1. B. Jaffe, W.R. Cook, H. Jaffe, Piezoelectric ceramics. (Academic Press, New York, 1971)

2. J. Rödel, K. G. Webber, R. Dittmer, W. Jo, M. Kimura, D. Damjanovic, J. Eur. Ceram. Soc. 35, $1659(2015)$

3. Y. Saito, H. Takao, T. Tani, T. Nonoyama, K. Takatori, T. Homma, T. Nagaya, M. Nakamura, Nature 432, 84 (2004)

4. D.A. Ochoa, J.E. García, R. Pérez, A. Albareda, IEEE Trans. Ultrason. Ferroelectr. Freq. Contr. 55, $2732(2008)$

5. J. E. Garcia, R. Perez, D. A. Ochoa, A. Albareda, M. H. Lente, J. A. Eiras, J. Appl. Phys. 103, $054108(2008)$

6. D. A. Ochoa, J. E. Garcia, I. Tamayo, V. Gomis, D. Damjanovic, R. Perez, J. Am. Ceram. Soc. 951656 (2012)

7. A. Safari, M. Abazari, K. Kerman, N. Marandian-Hagh, E. K. Akdoğan, IEEE Trans. Ultrason. Ferroelectr. Freq. Control 56, 1586 (2009)

8. D. A. Ochoa, J. E. Garcia, R. Perez, V. Gomis, A. Albareda, F. Rubio-Marcos, J. F. Fernandez, J. Phys. D: Appl. Phys. 42, 025402 (2009)

9. G. Robert, D. Damjanovic, N. Setter, Appl. Phys. Lett. 77, 4413 (2000)

10. S.A. Turik, L.A. Reznitchenko, A.N. Rybjanets, S.I. Dudkina, A.V. Turik, A.A. Yesis, J. Appl. Phys. 97, 64102 (2005)

11. G. Robert, D. Damjanovic, N. Setter, A.V. Turik, J. Appl. Phys. 89, 5067 (2001)

12. G. Robert, D. Damjanovic, N. Setter, J. Appl. Phys. 90, 2459 (2001) 
This is the post-print (i.e. final draft post-refereeing) of the publication.

The final publication is available at Springer via http://dx.doi.org/ 10.1007/s00339-016-9808-1

13. G. Bertotti, I. Mayergoyz, The Science of Hysteresis, vol 3.(Elsevier Academic Press, Oxford, 2006)

14. J. E. Garcia, R. Perez, A. Albareda, J. Phys.: Condens. Matter 17, 7143 (2005)

15. A.V. Turik, Sov. Phys. Solid State 5, 885 (1963)

16. D.V. Taylor, D. Damjanovic, J. Appl. Phys. 82, 1973 (1997)

17. D.A. Hall, J. Mater. Sci. 36, 4575 (2001)

18. J. E. Garcia, D. A. Ochoa, V. Gomis, J. A. Eiras, R. Perez, J. Appl. Phys. 112, 014113 (2012)

19. K. Carl, K. H. Härdtl, Ferroelectrics 17, 473 (1978)

20. A. Sutor, S.J. Rupitsch, R. Lerch, Appl. Phys. A 100, 425 (2010)

21. F. Wolf, A. Sutor, S.J. Rupitsch, R. Lerch, Sens. Actuator A 172, 245 (2011)

22. F. Wolf, A. Sutor, S.J. Rupitsch, R. Lerch, Sens. Actuator A 186, 223 (2012)

23. S.J. Rupitsch, F. Wolf, A. Sutor, R. Lerch, Acta Mech. 223, 1809 (2012)

24. J.E. Garcia, R. Perez, A. Albareda, J. Phys. D: Appl. Phys. 34, 3279 (2001) 